\title{
Rod lengthening with the magnetically controlled growing rod: factors influencing rod slippage and reduced gains during distractions
}

Authors: $\quad{ }^{\mathbf{1}}$ Jason Pui Yin Cheung, MBBS, MMedSc, FHKCOS, FHKAM,

FRCSEd,

${ }^{\mathbf{1}}$ Karen Kar Lum Yiu, MSc

${ }^{1}$ Dino Samartzis, DSc

${ }^{1}$ Kenny Kwan, FHKCOS, FHKAM, FRCSEd

${ }^{1,2}$ Boon-Beng Tan, MD, Ms Ortho

${ }^{1}$ Kenneth Man Chee Cheung, MBBS, MD, FRCS, FHKCOS, FHKAM

Affiliations: $\quad{ }^{1}$ Department of Orthopaedics and Traumatology, The University of Hong Kong, Pokfulam, Hong Kong, SAR, China

2 Department of Orthopaedics, University Malaysia Sarawak

(UNIMAS)

Abstract

Introduction: Ability to achieve successful MCGR distraction is crucial for gradual spine lengthening. Rod slippage has been described as a failure of internal magnet rotation leading to a slippage and an inability to distract the rod. However, its onset, significance and risk factors are currently unknown. In addition, how this phenomenon pertains to actual distracted lengths is unknown. Hence, the aims of this study are to identify the factors that are associated with rod slippage and to study the pattern of achieved length gain with a standard distraction methodology.

11 Methods: A total of 22 patients with MCGR implantation with at least 6 distraction episodes were prospectively studied. Patients with rod slippage occurring $\leq 6$ distraction episodes were considered early rod slippage while those $>6$ or have yet not slipped were grouped as late rod slippage. The association of parameters including body habitus, maturity status, age of implantation, total number of distractions, months of distraction from initial implantation, initial and postoperative Cobb angle, T1-T12, T1-S1, T5-T12 kyphosis, curve flexibility, fusion block

17 length, and distance between magnets in dual rods and between the magnets and apex of the 
1 curve with early or late onset of rod slippage was studied. Differences between expected and 2 achieved distraction lengths were assessed with reference to rod slippage episodes and rod 3 exchanges to determine any patterns of diminishing returns.

4 Results: Patients had mean age of 7.1 years at diagnosis with mean follow-up of 49.8 months. A 5 mean 32.4 distractions were performed per patient. Early rod slippage occurred in 14 patients 6 and late rod slippage occurred in 8 patients. Increased height, weight, body mass index, older 7 age, increased T1-12 and T1-S1 lengths, and less distance between magnets were significantly 8 associated with early rod slippage. Expected distraction lengths did not translate to achieved 9 distraction lengths. Reduced length gains were observed after achieving one-third of the 10 allowable distracted length in the first MCGR implanted. However, this rate returned to its 11 original baseline after rod exchange with another reduction observed after half of the total 12 achievable length was obtained in the second MCGR.

13 Conclusion: This is the first study to specifically analyze the impact of rod slippage on 14 distraction lengths and the risk factors associated with its onset and frequency. Increased body 15 habitus and reduced distance between internal magnets significantly influenced rod slippage 16 events. In addition, mismatching between expected and achieved distraction lengths was 17 illustrated. Diminishing returns in distracted length gains were only observed after a certain 18 usage of the MCGR rather than the number of distractions and overall length gain. 
Introduction

Traditional growing rods (TGRs) have previously been the gold-standard for management of early-onset scoliosis (EOS) because they can prevent curve deterioration while allowing for physiological spinal growth. ${ }^{1-3}$ However, TGRs require frequent surgeries to perform open manual distractions of the rods. As such, there is an increased risk of anesthestic and wound complications. ${ }^{4}$ A "law of diminishing returns" also occurs with TGR use in which the average T1-S1 gains from a surgical lengthening decreases with repeated lengthening procedures and occurs as early as the first lengthening procedure. ${ }^{5}$ The proposed mechanism for this phenomenon is progressive stiffness of spinal segments as a result of prolonged in-situ instrumentation or spine auto-fusion.

The development of magnetically controlled growing rods (MCGRs) has changed EOS management by adopting the use of a remotely distractible growing rod to allow for non-invasive and non-surgical means for spine lengthening. ${ }^{6,7}$ This allows for more frequent distractions, potentially mimicking normal physiological growth more closely, and for more cost-effective treatment of patients. More frequent and smaller amounts of distractions may also prevent or delay auto-fusion of the spine and by extension the law of diminishing returns. ${ }^{8}$ The MCGR's short-term clinical effectiveness have been reported, ${ }^{7,9-12}$ and its surgical indications can be extended to gradual correction of severe deformities as well. ${ }^{13}$ Despite the need for more regular imaging for monitoring the more frequent distractions, ultrasonic monitoring of distractions allows reduced radiation exposure with comparable accuracy to plain radiographs. ${ }^{14-16}$

With better understanding of the MCGR after years of use, several important complications have been identified such as distraction failure, proximal junctional kyphosis and rod slippage. 6 This rod slippage or "clunking phenomenon" manifests during distraction episodes as a palpable and audible "clunk". It may indicate a failure of distraction or slippage of the MCGR's internal mechanism in which the rod's magnet fails to complete a full revolution followed by stalling and a return to its initial position. ${ }^{6}$ Normally, distractions should be smooth with a "wobble" feeling of the external remote controller (ERC) characterized by smooth continuous internal magnet turning. ${ }^{6}$ Due to the failed magnet rotation, rod slippage may cause failure of the rod to lengthen the spine but without any manifestation of symptoms. Nevertheless, factors associated with its occurrence and its effect on achieved lengthening needs to be addressed, since it may influence the safety and effectiveness of MCGR. Currently there is no understanding on why it occurs and the significance of an early or late rod slippage. As such, the objective of this study is to analyze factors influencing rod slippage and its effects, primarily upon patterns of length gains with MCGR distraction.

\section{Methods}

This was a prospective study of consecutive EOS (scoliosis diagnosed earlier than 10 years of age) patients treated with the MCGR from December 2009 to March 2016. All patients with at least 2 years of follow-up and 6 distraction episodes were recruited. Ethics approval was obtained via the local institutional review board. All patients had substantial remaining growth potential (premenarche and Risser 0) prior to initial implantation. Preoperative flexibility was assessed by fulcrum-bending radiographs of which the methods for obtaining fulcrum-bending radiographs have been previously reported. ${ }^{17-20}$ All patients underwent standard implantation of 
the MCGR without any intraoperative rod distractions. Both single and dual MCGRs were included in this study. All dual rods were placed in a standard and offset rod configuration.

All distractions were performed by three experienced MCGR users. The first distraction was done one month postoperatively and followed thereafter on a monthly basis. An expected $2 \mathrm{~mm}$ distraction length was applied to the ERC for each distraction episode. The distraction was carried out fully on one rod before the other rod if no slippage occurred. The slippage of the magnetic mechanism was identified through a clunking sound and feeling during the distractive procedure. If rod slippage occurred, other distraction techniques, such as single magnet distraction, alternate distractions with smaller amounts (i.e. $0.5 \mathrm{~mm}$ alternating between rods), or alternative positioning of the patient would be performed to try and maximize the amount of distractions obtained.

Early-onset rod slippage was defined as a slippage occurring $\leq 6$ distractions and lateonset rod slippage was defined as slippage occurring $>6$ distractions or those that have never experienced slippage. Multiple parameters were studied as factors influencing the timing of rod slippage. These parameters included the maturity status as indicated by the Risser sign and distal radius and ulna (DRU) classification ${ }^{21-23}$, age of implantation, body height $(\mathrm{cm})$, weight $(\mathrm{kg})$ and body mass index (BMI: $\mathrm{kg} / \mathrm{m}^{2}$ ), total number of distractions, months of distraction from initial implantation, initial, fulcrum-bending and postoperative coronal Cobb angle, T1-T12 and T1-S1 distance, T5-T12 kyphosis, correction rate, fulcrum flexibility, fulcrum-bending corrective index (FBCI), instrumented segment length, and distance between magnets in dual rods and between the magnets and apex of the curve. Calculations for correction rate, fulcrum flexibility and FBCI $^{17-19}$ are as follows:

Correction rate $=$ (preoperative Cobb angle - immediate postoperative Cobb angle) / preoperative Cobb angle x 100\%

Fulcrum flexibility $=$ (preoperative Cobb angle - fulcrum-bending Cobb angle) $/$ preoperative Cobb angle x 100\%

FBCI = (preoperative Cobb angle - immediate postoperative Cobb angle) / (preoperative Cobb angle - fulcrum-bending Cobb angle) x 100\%

With the use of ultrasonic monitoring of distractions ${ }^{14-16}$, radiographs were only performed every six-months for assessment of Cobb angle and overall alignment. All images were digitized and imported into a digital imaging and medical software (RadWorks version 5.1) where the above parameters were measured. 
The trend of expected and achieved distraction lengths were plotted on a graph between the two rods. Expected distraction length indicated the amount of distraction length input into the ERC, which was a standardized $2 \mathrm{~mm}$ for all distraction episodes. Achieved distraction length indicated the actual length gain measured at the housing unit of the MCGR on radiographs. The differences between the expected and achieved lengths were also determined and compared with the amount of total length gained, when rod slippage occurred and when rod exchange was performed. The relationship of these factors would help explain the cause of any dissociation between expected and achieved distraction lengths.

\section{Statistical analysis}

All data were reported as mean \pm standard deviation (SD). Due to the use of radiographs for parameter measurements, expected and achieved distractions lengths were charted at every 6months to correlate. Relationships with total length distracted on the MCGR, rod slippage episodes, and timing of rod exchanges were charted. Analysis was performed by SPSS version 21 (Chicago, USA) and charts were created by Microsoft Excel. Factors influencing the onset of rod slippage episodes were analysed by Mann-Whitney tests. Significance was considered for pvalues $<0.05$.

\section{Results}

Twenty-two patients were included in this study. They had a mean age of $7.1 \pm 4.0$ years at diagnosis and 10.2 \pm 3.4 years at initial rod implantation with a mean follow-up of $49.8 \pm 11.0$ months and mean $32.4 \pm 11.0$ total distractions. The diagnoses included idiopathic $(n=15 ; 2$ of which were infantile), congenital $(n=1)$, syndromal $(n=4)$, and neurofibromatosis $(n=2)$. The mean height was $137.5 \pm 16.2 \mathrm{~cm}$, weight was $35.1 \pm 10.9 \mathrm{~kg}$ and $\mathrm{BMI}$ was $16.5 \pm 3.9 \mathrm{~kg} / \mathrm{m}^{2}$ at first rod implantation.

The mean preoperative, immediate postoperative and latest follow-up coronal Cobb angle was 56.2 \pm 10.3 degrees, 23.6 \pm 8.7 degrees and 27.9 \pm 11.4 degrees, respectively. The mean fulcrum bending Cobb angle was 17.5 \pm 11.6 degrees. This accounted for a mean correction rate of $57.1 \pm 17.6 \%$, fulcrum flexibility of $69.0 \pm 22.7 \%$ and FBCI of $89.4 \pm 20.2 \%$. The mean T5-12 kyphosis was $27.2 \pm 19.6$ degrees preoperatively, 17.6 \pm 13.0 degrees at immediate postoperative and 24.0 \pm 19.4 degrees at final follow-up.

The mean T1-12 length was $203.7 \pm 27.9 \mathrm{~mm}$ preoperatively, $211.6 \pm 24.4 \mathrm{~mm}$ immediate postoperatively, and $235.7 \pm 33.7 \mathrm{~mm}$ at final follow-up. The mean T1-S1 length was $333.3 \pm 43.1 \mathrm{~mm}$ preoperatively, $350.7 \pm 39.8-\mathrm{mm}$ immediate postoperatively, and $380.6 \pm 45.5 \mathrm{~mm}$ at final follow-up. The mean distance between magnets was $44.4 \pm 8.7 \mathrm{~mm}$ and mean distance between magnet and apical vertebrae was $45.0 \pm 36.1-\mathrm{mm}$ and $49.4 \pm 31.7 \mathrm{~mm}$ for the right and left magnets, respectively. 
Of the 22 patients, 14 had early-onset rod slippage and 8 had late-onset rod slippage. The mean age at diagnosis for early slippage patients was 8.6 \pm 3.9 years and for late slippage was $4.8 \pm 2.8$ years. The mean onset of slippage for the early group was $2.2 \pm 1.5$ and $3.7 \pm 2.8$ for the right and left rods, respectively. The mean onset of slippage for the late group was $10.2 \pm 2.6$ and 19.5 \pm 12.7 for the right and left rods, respectively. Body habitus (weight, height and BMI), older age (higher DRU grade and chronological age) and increased preoperative and postoperative T112 and T1-S1 were significant risk factors for early rod slippage (Table 1).

Mean deviation between expected and achieved distraction lengths were $-8.4 \pm 7.3 \mathrm{~mm}$ and $-5.6 \pm 5.4 \mathrm{~mm}$ for the right and left rods, respectively. The mean total actual distracted length before onset of rod slippage was $9.9 \pm 7.8 \mathrm{~mm}$ for the right rod and $14.5 \pm 12.6 \mathrm{~mm}$ for the left rod. Rod slippage occurred earlier after the first rod exchange with mean $17.5 \pm 17.1 \mathrm{~mm}$ and $23.6 \pm 27.0 \mathrm{~mm}$ actual distracted length before onset of rod slippage for the right and left first rod, respectively; and mean $5.2 \pm 7.3 \mathrm{~mm}$ and $7.3 \pm 7.2 \mathrm{~mm}$ actual distracted length before onset of rod slippage for the right and left second rod, respectively. There was maximum of 2 rod exchanges per patient and none of the third rods have experienced rod slippage thus far in our follow-up. There was a mean $18.9 \pm 10.3$ distractions of the right rod and a mean $19.4 \pm 11.1$ distractions of the left rod prior to rod exchange. This amounted to $27.0 \pm 10.6 \mathrm{~mm}$ and $32.1 \pm 6.5 \mathrm{~mm}$ distracted length before rod exchange.

Expected distraction lengths did not translate to achieved distraction lengths (Figure 1) through time suggesting that the deviation between what we expected to achieve and what length was actually distracted increased with the number of distractions. As illustrated in Figure 2, the achieved distraction length reduced after approximately 12 months of postoperative follow-up, which translated to approximately $14-16 \mathrm{~mm}$ total achieved lengthening. Furthermore, similar reduction of achieved length gain was observed at 36 months, which was approximately 12 months after the first rod exchanges that occurred at 24 months of follow-up. Observing the early and late rod slippage groups separately, those with early slippage appeared to require increased expected lengthening to achieve similar actual lengthening as the late slippage group (Figure 3a). This translated to increased mismatch between the expected and achieved distraction lengths (Figure 3b).

\section{Discussion}

The MCGR is unquestionably a significant advancement for managing EOS. Despite its recognized benefits of allowing outpatient distractions without open surgeries under general anesthesia, long-term experience with this device is lacking. ${ }^{711}$ Of the reported complications, the significance of rod slippage is of great interest. Early slippage may be an indicator of a possible flaw in the design of the distraction device or the magnetic mechanism. Alternatively, it may be regarded as a physiological protective mechanism to prevent overdistraction beyond what the spine can tolerate. Nevertheless, rod slippage appears to be associated with the high resistance or counter-force during distractions. At this limit, the rod is unable to impart enough force to allow for lengthening; thereby, leading to distraction failure. 
The main study objective was to determine whether rod slippage prevents achieving desired distraction length, thus interfering with the balance between curve correction and truncal growth. Hence, it was important to determine whether occurrences of rod slippage episodes coincided with increased deviations between expected and achieved distraction lengths. This can be observed with the increasing mismatches between expected and achieved length gains for the early rod slippage groups (Figure 3). Although the overall achieved gains were similar, this is likely due to the authors' practice of modifying the distraction techniques to improve the likelihood of successful length gain. These techniques include using a single magnet distraction with alternating smaller amounts of distraction and different patient positioning such as sitting forward. This may help to reduce the degree of force required to lengthen per distraction and to make the rod more pronounced for magnetic attraction by the ERC, respectively. The position of the ERC is crucial to achieving satisfactory length gains and should be addressed in future study. Methods such as placing the actuator in between the two magnets of the external device, placing only a single magnet over the actuator or placing one magnet on each actuator if two standard rods are used may affect rod slippage rates as the induced forces may differ. ${ }^{6}$

Several specific risk factors that may influence the timing and onset of rod slippage were studied. In addition to body habitus, age, curve magnitude, spine length and alignment, we also studied the distance between internal magnets of the 2 rods or cross-talk and magnets too close to the apex of the major curve. One of the laws in magnetism states that the force of attraction or repulsion between two magnetic poles is directly proportional to the product of the strengths of the poles and inversely proportional to the square of the distance between them. Therefore, a shorter distance between the internal magnets will lead to a greater force and influence on each other. In other words, the chances of rod slippage in both MCGR are expected to be higher when the distance between the internal magnets are shorter. This was observed in our study as those with approximately $1 \mathrm{~cm}$ less distance between magnets was associated with early rod slippage. Magnets too close to the apex, which is usually the stiffest part of the curve, may also contribute to earlier rod slippage. In such situations, more force may be required to counter the internal resistance. In this study however, no significant differences were observed between groups.

Another major contributor of early rod slippage was increased body habitus (weight, height and BMI). Other significant parameters such as chronological age, maturity (radius and ulna grades), T1-T12, T1-S1 lengths are part of the profile of larger sized patients. These patients will usually have a thicker layer of soft tissue between the external and internal magnets, thereby reducing the amount of force subjected onto the internal magnet to produce a full revolution. It is of note that the authors do not perform intraoperative distractions of the rods, have a standardized time of first distraction from implantation, and intended distraction length per episode. The timing of first rod slippage from initial rod implantation may be related to patients' intrinsic soft tissue properties and response to viscoelasticity. Hence, for future studies, the effect of soft tissue tension and its response to distraction forces should be addressed. In addition, it is of interest to determine the limit of soft tissue thickness between the ERC and internal magnet for accomodating smooth distractions without slippage. 
This study adopts a systematic assessment of increasing deviations in expected and achieved distraction lengths (Figure 1) to observe for their relationships with rod slippage episodes and rod exchanges. The standard MCGR design allows for 48mm total distractable length. At approximately one-third of this length distracted $(\sim 14-16 \mathrm{~mm})$ as seen in Figure 2, deviations in expected and achieved distraction lengths were observed with reduced achieved length gains per distraction. Further rod distractions are henceforth more difficult to achieve comparable lengths. However, this phenomenon only occurs for the same rod undergoing distraction. Once the rod has been exchanged, distractions are once again matching well between expected and achieved length gains until again the rod length reaches half of the maximum allowable length. Hence, this explains previous reports of reducing length gains with distractions. ${ }^{24,25}$ These studies adopt a more liberal amount of distraction length per distraction and thus deviation of expected and achieved length gains occur earlier.

With longer follow-up and taking into account the effect of rod exchanges, it is likely that better matching of expected and achieved gains may be observed. It is important to note that these findings may not translate to reduced T1-S1 gains. Consistent T1-S1 gains with occasional interval reductions in the MCGR has been reported with a monthly distraction protocol. ${ }^{8}$ This finding suggests that the original description of the "law of diminishing returns" related to the $\mathrm{TGR}^{5}$ is not fully applicable to the MCGR as T1-S1 gains are consistent. Our findings suggest that the "diminishing returns" relates to increasing distracted amounts within each individual MCGR rather than the overall distracted spine length gained and the number of distractions. As such, after a rod exchange, achieved gains were again as successful as the initial findings with the first MCGR. This may be a limitation within the current rod designs. The relationship between T1-S1 gains and actual rod distraction length gains should be further studied. In this series, it appears that most of the T1-12 and T1-S1 gains are achieved in the first surgery with curve correction but gains are observed at the final follow-up.

The limitations of this study include the heterogeneity of the subjects and the small sample size. Although our study represents a small sample size, this is attributed to the recent introduction of MCGR in EOS patients. In time and in the establishment of a multicenter network of MCGR sites, larger sample sizes can be gathered to faciliate more extensive statistical analyses. Nonetheless, our study is the first to specifically address rod slippage and its significance with distractions. A possible rationale for the high occurrence in early slippage in our study is an older population (mean 10.2 years) at initial rod implantation. This may be related to our intent and success in treating our EOS patients with bracing as their mean age of diagnosis is 3 years earlier. Hence, it is of interest to see if similar conclusions can be drawn from a younger EOS population. Finally, our report is only short to mid-term follow-up. Long-term follow-up is necessary to determine whether our findings of “diminishing returns" pertains only to the remaining length of individual rods rather than prolonging distraction episodes.

\section{Conclusions}


This is the first study to analyze this rod slippage phenomenon with distractions in EOS 2 patients with MCGR. The MCGR is an evolution from the TGR for managing scoliosis in 3 skeletally immature children. Despite the advancement in technology and its obvious advantages 4 compared to traditional methods, our knowledge is still limited. This study is important 5 nonetheless to raise awareness for rod slippage events and the importance of interval 6 measurements of expected and achieved rod length gains. The appearance of rod slippage should 7 not deter clinicians from modifying the distraction technique and patient positioning in an 8 attempt to achieve more satisfactory results. Its occurrence may be related to older aged and 9 larger-sized patients with smaller distance between internal magnets, but does not significantly 10 reduce achievable length with distractions. The "law of diminishing returns", unlike TGR, does 11 not occur with each subsequent distractions but rather, is specific to the amount of lengthening 12 already achieved per MCGR. Consistent length gains are still observed and diminished returns 13 are only observed after more than half of the rod's available length is achieved. Further larger 14 scale clinical and biomechanical studies are necessary to further understand and validate our 15 results. 


\section{References}

3 [1]. Akbarnia BA, Breakwell LM, Marks DS, McCarthy RE, Thompson AG, Canale SK, 4 Kostial PN, Tambe A, Asher MA, Growing Spine Study G. Dual growing rod technique 5 followed for three to eleven years until final fusion: the effect of frequency of lengthening. Spine 6 (Phila Pa 1976) 2008;33:984-90.

7 [2]. Akbarnia BA, Marks DS, Boachie-Adjei O, Thompson AG, Asher MA. Dual growing 8 rod technique for the treatment of progressive early-onset scoliosis: a multicenter study. Spine 9 (Phila Pa 1976) 2005;30:S46-57.

10 [3]. Winter RB, Moe JH, Lonstein JE. Posterior spinal arthrodesis for congenital scoliosis. An Surg Am 1984;66:1188-97.

[4]. Akbarnia BA, Emans JB. Complications of growth-sparing surgery in early onset scoliosis. Spine (Phila Pa 1976) 2010;35:2193-204.

[5]. Sankar WN, Skaggs DL, Yazici M, Johnston CE, 2nd, Shah SA, Javidan P, Kadakia RV, Day TF, Akbarnia BA. Lengthening of dual growing rods and the law of diminishing returns. Spine (Phila Pa 1976) 2011;36:806-9.

[6]. Cheung JP, Cahill P, Yaszay B, Akbarnia BA, Cheung KM. Special article: Update on the magnetically controlled growing rod: tips and pitfalls. J Orthop Surg (Hong Kong) 2015;23:383-90.

[7]. Cheung KM, Cheung JP, Samartzis D, Mak KC, Wong YW, Cheung WY, Akbarnia BA, Luk KD. Magnetically controlled growing rods for severe spinal curvature in young children: a prospective case series. Lancet 2012;379:1967-74.

[8]. Cheung JP, Bow C, Samartzis D, Kwan K, Cheung KM. Frequent small distractions with a magnetically controlled growing rod for early-onset scoliosis and avoidance of the law of diminishing returns. J Orthop Surg (Hong Kong) 2016;24:332-7.

[9]. Akbarnia BA, Pawelek JB, Cheung KM, Demirkiran G, Elsebaie H, Emans JB, Johnston CE, Mundis GM, Noordeen H, Skaggs DL, Sponseller PD, Thompson GH, Yaszay B, Yazici M, Growing Spine Study G. Traditional Growing Rods Versus Magnetically Controlled Growing Rods for the Surgical Treatment of Early-Onset Scoliosis: A Case-Matched 2-Year Study. Spine Deform 2014;2:493-7.

[10]. Figueiredo N, Kananeh SF, Siqueira HH, Figueiredo RC, Al Sebai MW. The use of magnetically controlled growing rod device for pediatric scoliosis. Neurosciences (Riyadh) 2016;21:17-25.

[11]. Hosseini P, Pawelek J, Mundis GM, Yaszay B, Ferguson J, Helenius I, Cheung KM, Demirkiran G, Alanay A, Senkoylu A, Elsebaie H, Akbarnia BA. Magnetically controlled Growing Rods for Early-onset Scoliosis: A Multicenter Study of 23 Cases With Minimum 2 years Follow-up. Spine (Phila Pa 1976) 2016;41:1456-62.

[12]. Cheung KM, Cheung JP, Samartzis D. Magnetically controlled growing rods for scoliosis in children - Authors' reply. Lancet 2012;380:1228-9.

[13]. Cheung JP, Samartzis D, Cheung KM. A novel approach to gradual correction of severe spinal deformity in a pediatric patient using the magnetically-controlled growing rod. Spine $J$ 2014;14:e7-13. 
[14]. Cheung JP, Bow C, Samartzis D, Ganal-Antonio AK, Cheung KM. Clinical utility of ultrasound to prospectively monitor distraction of magnetically controlled growing rods. Spine $J$ 2016;16:204-9.

[15]. Cheung JP, Yiu KK, Bow C, Cheung PW, Samartzis D, Cheung KM. Learning Curve in Monitoring Magnetically Controlled Growing Rod Distractions With Ultrasound. Spine (Phila Pa 1976) 2017;

[16]. Stokes OM, O'Donovan EJ, Samartzis D, Bow CH, Luk KD, Cheung KM. Reducing radiation exposure in early-onset scoliosis surgery patients: novel use of ultrasonography to measure lengthening in magnetically-controlled growing rods. The spine journal : official journal of the North American Spine Society 2014;14:2397-404.

[17]. Cheung KM, Luk KD. Prediction of correction of scoliosis with use of the fulcrum bending radiograph. J Bone Joint Surg Am 1997;79:1144-50.

[18]. Luk KD, Cheung WY, Wong Y, Cheung KM, Wong YW, Samartzis D. The predictive value of the fulcrum bending radiograph in spontaneous apical vertebral derotation in adolescent idiopathic scoliosis. Spine (Phila Pa 1976) 2012;37:E922-6.

[19]. Shigematsu H, Cheung JP, Bruzzone M, Matsumori H, Mak KC, Samartzis D, Luk KD. Preventing Fusion Mass Shift Avoids Postoperative Distal Curve Adding-on in Adolescent Idiopathic Scoliosis. Clin Orthop Relat Res 2017;475:1448-60.

[20]. Yao G, Cheung JP, Shigematsu H, Ohrt-Nissen S, Cheung KM, Luk KD, Samartzis D. Characterization and Predictive Value of "Segmental Curve Flexibility" in Adolescent Idiopathic Scoliosis Patients. Spine (Phila Pa 1976) 2016;

[21]. Cheung JP, Cheung PW, Samartzis D, Cheung KM, Luk KD. The use of the distal radius and ulna classification for the prediction of growth: peak growth spurt and growth cessation. Bone Joint J 2016;98-B:1689-96.

[22]. Cheung JP, Samartzis D, Cheung PW, Cheung KM, Luk KD. Reliability Analysis of the Distal Radius and Ulna Classification for Assessing Skeletal Maturity for Patients with Adolescent Idiopathic Scoliosis. Global Spine J 2016;6:164-8.

[23]. Cheung JP, Samartzis D, Cheung PW, Leung KH, Cheung KM, Luk KD. The distal radius and ulna classification in assessing skeletal maturity: a simplified scheme and reliability analysis. J Pediatr Orthop B 2015;24:546-51.

[24]. Lebon J, Batailler C, Wargny M, Choufani E, Violas P, Fron D, Kieffer J, Accadbled F, Cunin V, De Gauzy JS. Magnetically controlled growing rod in early onset scoliosis: a 30-case multicenter study. Eur Spine J 2016;

[25]. Rolton D, Thakar C, Wilson-MacDonald J, Nnadi C. Radiological and clinical assessment of the distraction achieved with remotely expandable growing rods in early onset scoliosis. Eur Spine J 2016;25:3371-6. 
2 Table 1: Parameters leading to slippage episodes

\begin{tabular}{|c|c|c|c|c|}
\hline Parameter at implantation & $\begin{array}{l}\text { Mean } \pm \text { SD } \\
(n=22)\end{array}$ & $\begin{array}{l}\text { Mean } \pm \text { SD } \\
\text { (early slippage, } \\
n=14 \text { ) }\end{array}$ & $\begin{array}{l}\text { Mean } \pm \text { SD } \\
\text { (no or late } \\
\text { slippage, } n=8 \text { ) }\end{array}$ & p-value \\
\hline Height $(\mathrm{cm})$ & $137.5 \pm 16.3$ & $146.4 \pm 12.2$ & $106.4 \pm 8.5$ & $0.001^{*}$ \\
\hline Weight (kg) & $30.0 \pm 11.2$ & $35.6 \pm 10.2$ & $17.7 \pm 2.5$ & $0.001^{*}$ \\
\hline $\operatorname{BMI}\left(\mathrm{kg} / \mathrm{m}^{2}\right)$ & $14.9 \pm 4.7$ & $15.4 \pm 5.8$ & $12.0 \pm 1.7$ & $0.006 *$ \\
\hline Risser sign & $0.4 \pm 0.9$ & $0.6 \pm 1.0$ & $0.0 \pm 0.0$ & 0.104 \\
\hline Radius grade & $5.4 \pm 1.4$ & $6.2 \pm 0.9$ & $3.8 \pm 1.0$ & $0.007^{*}$ \\
\hline Ulna grade & $3.9 \pm 1.8$ & $5.0 \pm 1.0$ & $2.1 \pm 1.3$ & $0.006 *$ \\
\hline Chronological age (years) & $10.2 \pm 3.4$ & $12.1 \pm 1.9$ & $7.2 \pm 2.9$ & $0.003 *$ \\
\hline Cobb angle (degrees) & $56.2 \pm 10.3$ & $57.6 \pm 13.0$ & $60.0 \pm 12.0$ & 0.124 \\
\hline Fulcrum Cobb angle (degrees) & $17.5 \pm 11.6$ & $18.6 \pm 12.1$ & $14.8 \pm 11.2$ & 0.843 \\
\hline Fulcrum flexibility (\%) & $69.0 \pm 22.7$ & $69.3 \pm 19.4$ & $60.1 \pm 28.7$ & 0.843 \\
\hline $\mathrm{T} 1-12(\mathrm{~mm})$ & $203.7 \pm 27.9$ & $210.5 \pm 35.4$ & $185.2 \pm 14.2$ & $0.012 *$ \\
\hline $\mathrm{T} 1-\mathrm{S} 1(\mathrm{~mm})$ & $333.3 \pm 43.1$ & $347.0 \pm 50.8$ & $298.3 \pm 22.0$ & $0.003^{*}$ \\
\hline T5-12 kyphosis (degrees) & $27.2 \pm 19.6$ & $27.2 \pm 17.7$ & $32.5 \pm 25.3$ & 0.785 \\
\hline \multicolumn{5}{|l|}{ Parameters after implantation } \\
\hline Correction rate (\%) & $57.1 \pm 17.6$ & $58.5 \pm 13.3$ & $56.2 \pm 22.7$ & 0.838 \\
\hline FBCI (\%) & $89.4 \pm 20.2$ & $88.5 \pm 21.1$ & $79.6 \pm 17.7$ & 0.606 \\
\hline $\begin{array}{l}\text { Immediate postoperative Cobb } \\
\text { angle (degrees) }\end{array}$ & $23.6 \pm 8.7$ & $23.9 \pm 8.6$ & $25.0 \pm 9.9$ & 0.633 \\
\hline $\begin{array}{l}\text { Immediate postoperative T1- } \\
12(\mathrm{~mm})\end{array}$ & $211.6 \pm 24.4$ & $219.9 \pm 26.2$ & $194.0 \pm 14.4$ & $0.012 *$ \\
\hline $\begin{array}{l}\text { Immediate postoperative T1- } \\
\text { S1 (mm) }\end{array}$ & $350.7 \pm 39.8$ & $368.4 \pm 38.2$ & $317.5 \pm 17.2$ & $0.001 *$ \\
\hline $\begin{array}{l}\text { Immediate postoperative T5- } \\
12 \text { kyphosis (degrees) }\end{array}$ & $17.6 \pm 13.0$ & $18.9 \pm 12.8$ & $14.4 \pm 13.2$ & 0.765 \\
\hline Instrumented length (mm) & $228.0 \pm 38.4$ & $223.5 \pm 46.5$ & $232.5 \pm 16.7$ & 0.539 \\
\hline $\begin{array}{l}\begin{array}{l}\text { Distance between magnets } \\
(\mathrm{mm})\end{array} \\
\end{array}$ & $44.4 \pm 8.7$ & $40.4 \pm 7.9$ & $49.3 \pm 9.6$ & $0.022 *$ \\
\hline $\begin{array}{l}\text { Distance between right magnet } \\
\text { and curve apex (mm) }\end{array}$ & $45.0 \pm 36.1$ & $35.1 \pm 37.0$ & $57.4 \pm 33.0$ & 0.091 \\
\hline $\begin{array}{l}\text { Distance between left magnet } \\
\text { and curve apex (mm) }\end{array}$ & $49.4 \pm 31.7$ & $48.5 \pm 20.6$ & $49.2 \pm 43.4$ & 0.426 \\
\hline
\end{tabular}

* indicated statistical significance

4 Early slippage indicates a slippage $\leq 6$ distractions. Late slippage indicates no slip or slippage $>6$

5 distractions

6 BMI: body mass index; DRU: distal radius and ulna classification; FBCI: fulcrum bending

7 corrective index; SD: standard deviation 
Figure legends

2 Figure 1: Changes in expected versus achieved distraction lengths (mm) for the (a) left rod and

3 (b) right rod with follow-up. No significant relationship is observed between deviations of

4 expected and achieved distraction lengths and onset of rod slippage.

5 Figure 2: Change in rates of achieved lengthening $(\mathrm{mm})$ charted against the total achieved 6 length (mm) per rod through follow-up. Number of rod exchanges and when they occurred are 7 also charted. Results show that that reduced length gains are obtained occur after $~ 50 \%$ of the 8 rod total length has been obtained but the increased length gains return after rod exchange.

9 Figure 3: (a) Length gains between early and late onset rod slippage groups. The accumulated 10 achieved length gains for the early onset rod slippage group required a greater expected length 11 gain to achieve similar actual length gains as the late rod slippage group. (b) Hence, there is an 12 increase in mismatch between expected and achieved distraction lengths with the early rod 13 slippage group.

14

15

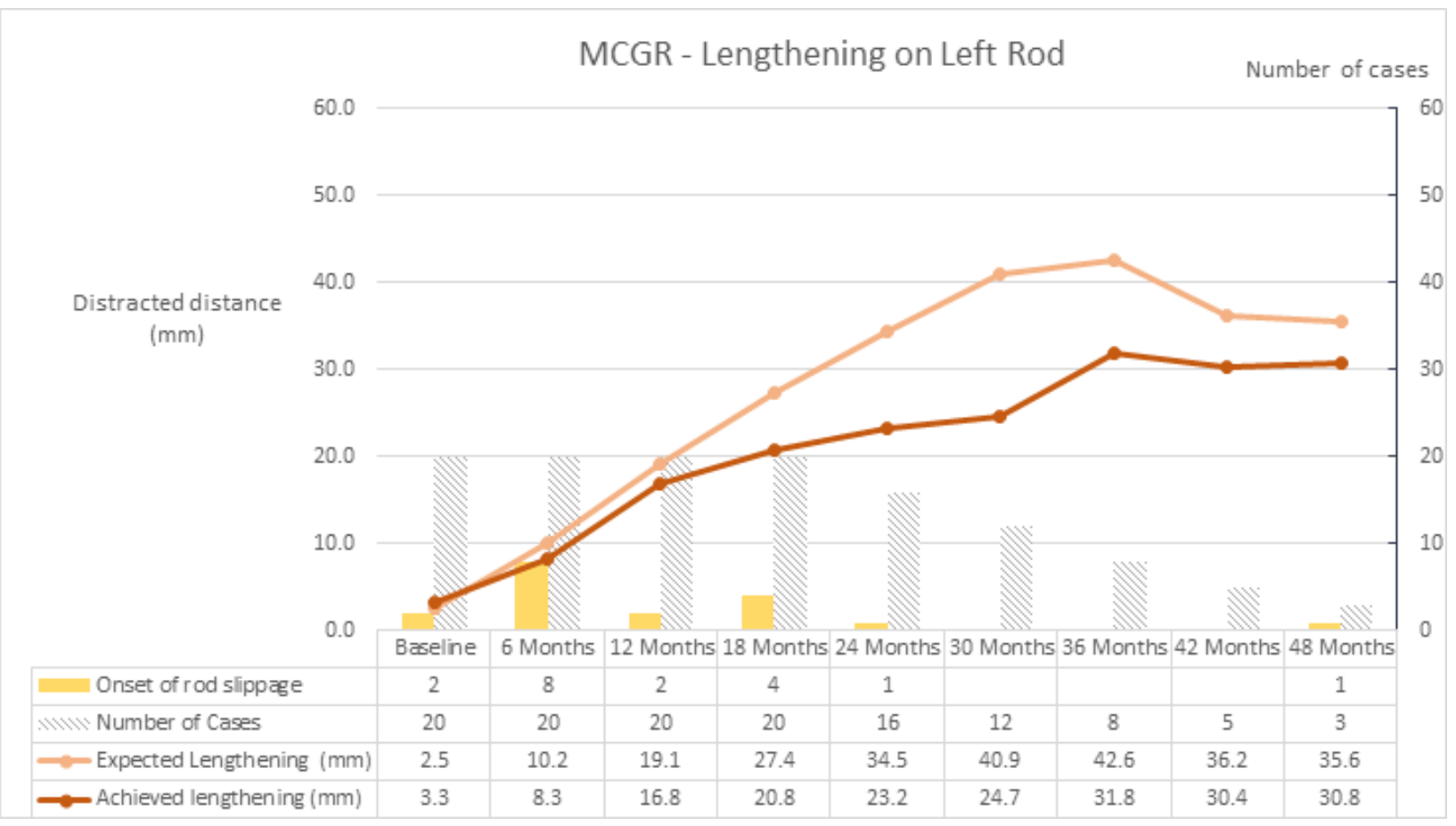

17 

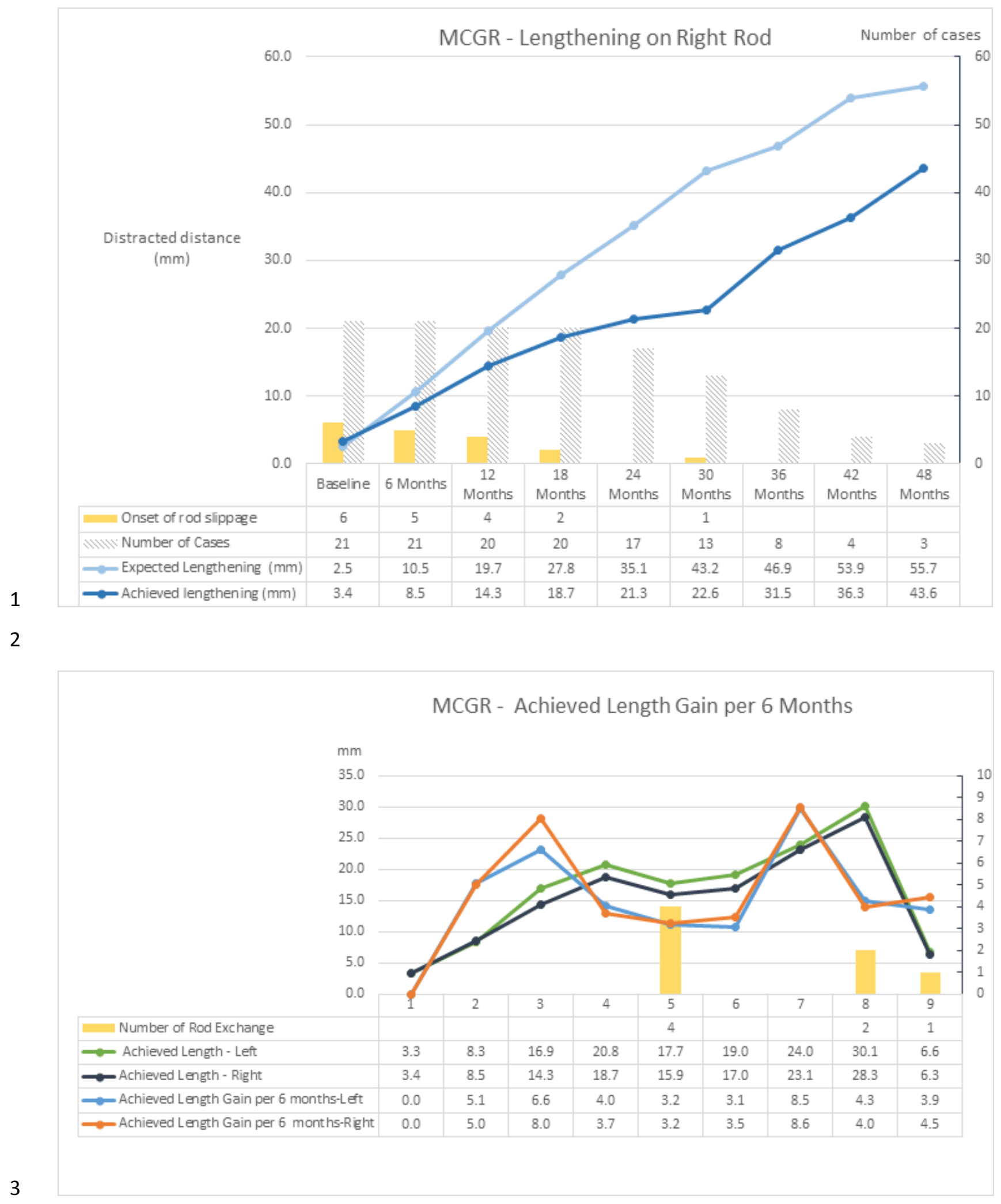


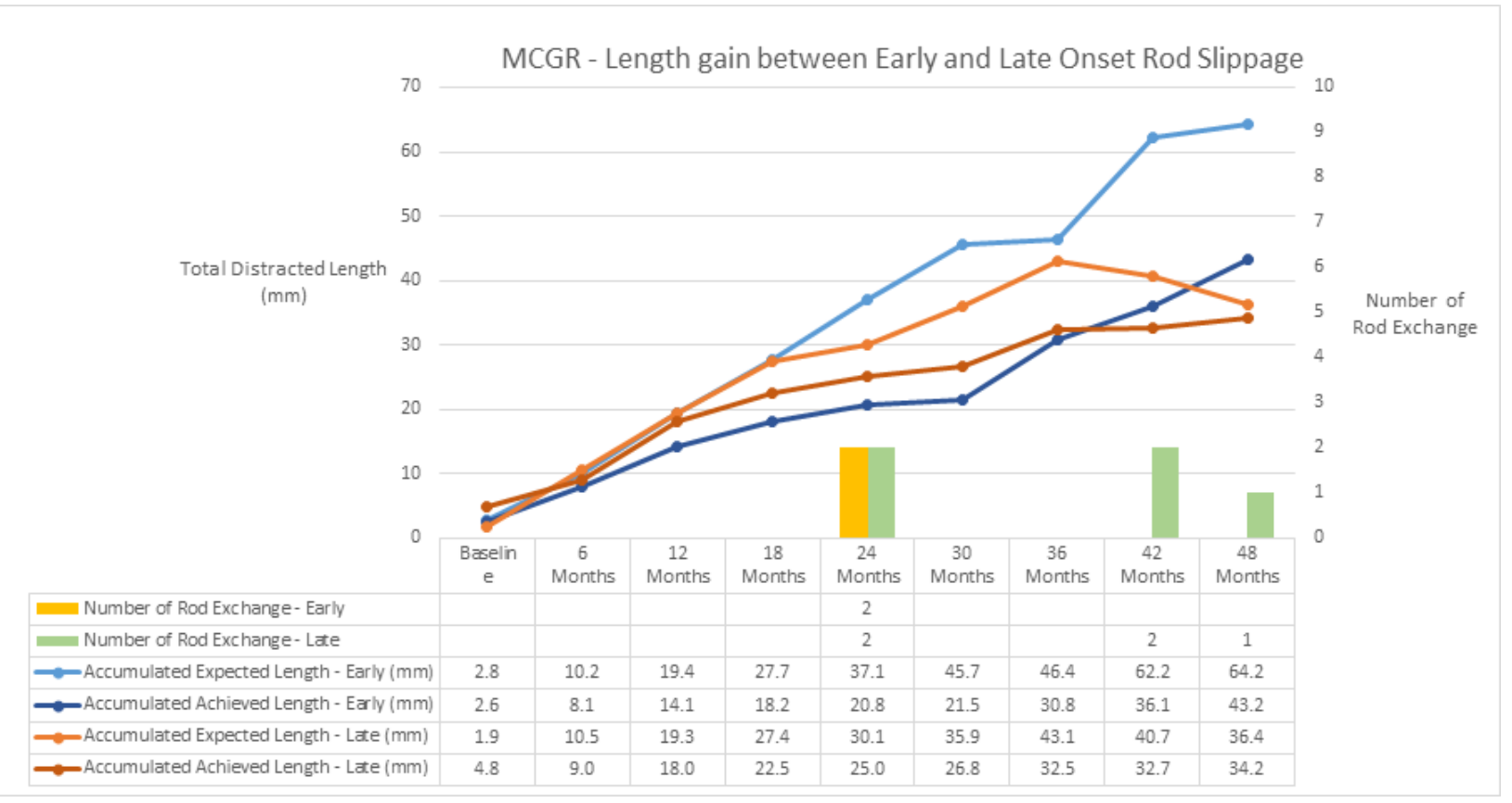

MCGR - Mismatch in Expected and Achieved Distraction Lengths with Timing of Rod Slippage

30

10

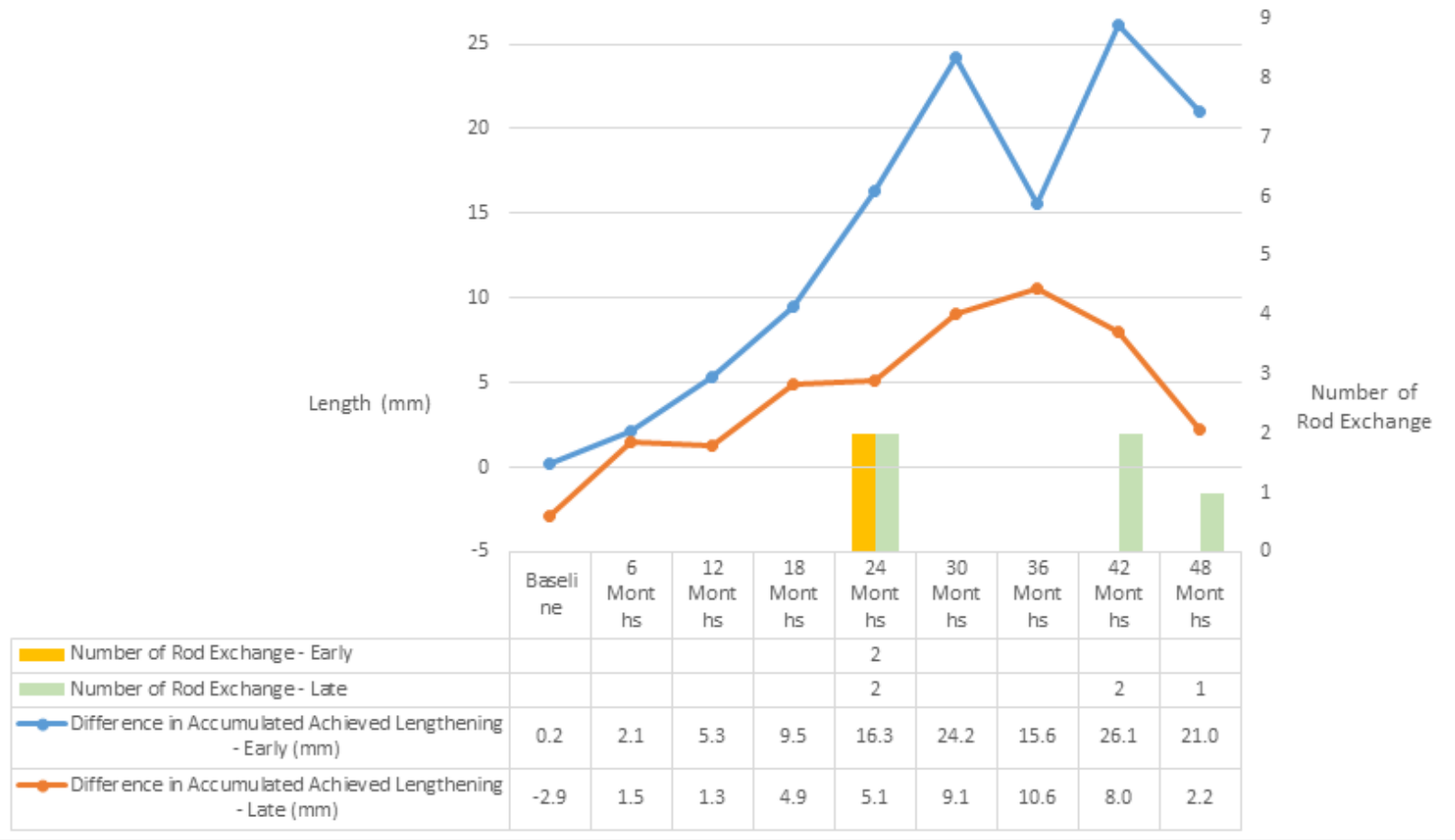

